\title{
DOCUMENTATION
}

\section{Une machine à glace pour équipes mobiles d'immunisation}

\author{
par A. LALANNE †
}

Depuis la création, déjà ancienne, des équipes mobiles d'immunisation, médecins et vétérinaires se sont efforcés de résoudre le problème de leur ravitaillement en glace. En effet la plupart des vaccins utilisés en médecine, tant humaine que vétérinaire, ne se conservent bien que sous le régime du froid et d'autre part, pour être efficace, l'équipe mobile doit avoir une très grande autonomie. Or cette autonomie est en fonction directe de l'approvisionnement en glace, celle-ci provenant de l'extérieur ou étant produite sur place au fur et à mesure des besoins.

Dans le premier cas des véhicules doivent être spécialement affectés au transport de glace. C'est une formule très onéreuse car au fur et à mesure que l'équipe s'éloigne, la dépense de transport de glace augmente et devient rapidement prohibitive. Certes, la mise en service de containers isothermes bien étudiés permet de limiter le nombre de voyages, mais on ne peut dépasser certaines limites dans la durée de la conservation de la glace, qui sont de l'ordre de 3 à 8 jours, suivant les régions et la température ambiante. Il s'ensuit une usure prématurée des véhicules et des dépenses très élevées, grevant lourdement les budgets et réduisant finalement le nombre des interventions.

C'est la raison pour laquelle chacun s'est ingénié à doter les équipes mobiles d'immunisation du matériel nécessaire à la production sur place de cette indispensable glace. Dans ce but, les appareils les plus divers ont été utilisés. Citons :

1. Les réfrigérateurs à absorption, encore largement employés, faute de mieux. Cependant, malgré les progrès réalisés au cours de ces dernières années, les modèles transportables présentent de nombreux inconvénients : production d'une faible quantité de glace par cycle, fragilité de certains éléments qui résistent mal aux déplacements en terrains variés, fonctionnement défectueux lorsque la température ambiante dépasse $30^{\circ}$, etc. Il existe actuellement des modèles produisant des quantités importantes de glace ( $10 \mathrm{~kg}$ et plus par jour) ; malheureusement leur poids, leur encombrement et leur fragilité rendent les déplacements répétés en tous terrains difficiles et délicats.

2. Les machines à glace à absorption (du type Ice-boy), moins fragiles et plus légères que les réfrigérateurs. Outre que la quantité de glace par cycle est très faible $(500$ à $700 \mathrm{~g}$ en 2 à 3 heures), ces appareils fonctionnent très mal — ou pas du tout - au-dessus de $30 / 35^{\circ}$. C'est ainsi que dans la zone sahélienne du Soudan Français on ne peut obtenir de petites quantités de glace (200 à $300 \mathrm{~g}$ par cycle) qu'en les faisant fonctionner pendant les heures les moins chaudes de la nuit.

Elles ont néanmoins rendu beaucoup de services et permettent aux équipes mobiles d'intervenir dans des régions encore interdites aux véhicules tous terrains.

3. Les bouteilles de gaz carbonique liquide qui, grâce à un détendeur spécialement étudié, permettent d'obtenir, en moins de 5 minutes, une galette de neige carbonique pesant environ $100 \mathrm{~g}$, suffisante pour une journée de conservation. On peut du reste répéter l'opération autant de fois qu'il est nécessaire. Ce procédé a été étudié et mis au point par le chef du Service. de l'Elevage de la Mauritanie en 1956. Il a toutefois signalé que l'obtention de neige carbonique est impossible lorsque la température de l'air ambiant dépasse $31^{\circ}$. On doit alors préalablement refroidir la bouteille, soit à l'aide d'enveloppes mouillées soumises à l'évaporation, soit par évaporation préalable d'un faible volume de gaz carbonique liquide. Mais dans ces conditions le rendement est très faible et 
on ne peut obtenir plus de 5 à 6 galettes par bouteille.

Si certaines précautions doivent être prises dans le transport et les manipulations des bouteilles, l'opération est cependant sans danger. Le procédé est élégant, rapide et présente d'incontestables avantages sur les appareils à absorption. Cependant le ravitaillement cn merce, même aux U.S.A., alors que les réfrigérateurs électriques à compresseur, de modèles très variés et de plus en plus perfectionnés, sont extrêmement nombreux sur le marché.

A ma connaissance, de telles machines à glace n'existent encore qu'à l'état de prototypes, dont trois ont été construits sur mes indications (prototype $\mathrm{n}^{\mathrm{0}} 1$ à Dakar et $\mathrm{n}^{\text {nis }} 2$ et 3 dans la

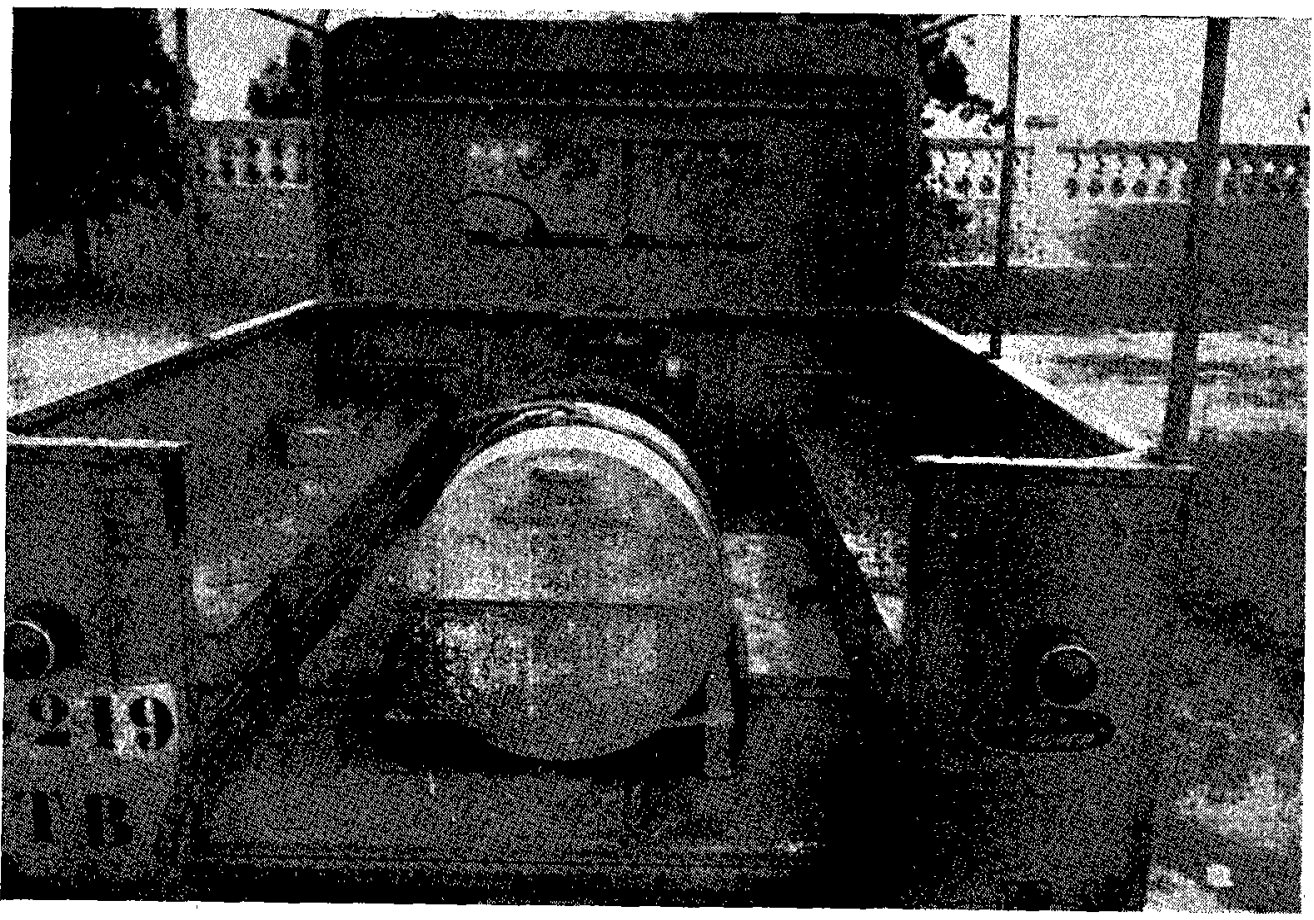

1. (Cliché A. Lalanne.) - L’appareil à glace sur pick-up Land-Rover, bloqué avec deux morceaux de madriers.

bouteilles de gaz carbonique exige de nombreux déplacements du véhicule de l'équipe mobile et, par ailleurs, l'approvisionnement en bouteilles de gaz carbonique liquide des postes de brousse éloignés pose des problèmes difficiles à résoudre. D'autre part, le prix de revient de la neige carbonique, de plus en plus élevé au fur et à mesure qu'on s'éloigne du centre de production du gaz carbonique liquide, est un élément dont il convient de tenir compte et, enfin, au-dessus d'une température ambiante de $+31^{\circ} \mathrm{C}$, la production de neige carbonique est, sinon impossible, du moins d'un prix de revient réellement prohibitif.

4. Les machines à glace à compresseur, l'énergie étant fournie par un petit moteur auxiliaire à essence. Il y a lieu de noter toutefois que de tels appareils n'existent pas encore dans le com-
Métropole). Pour les deux premiers, le principe était d'adapter un groupe compresseur-condenseur aux besoins particuliers d'une équipe mobile, l'entrainement du groupe étant réalisé au moyen d'un moteur à essence.

Le prototype $\mathrm{n}^{\circ} 2$, construit par les établissements Zhendre (1), plus puissant que le premier,'pour lequel avait été retenu un groupe compresseur-condenseur équipant les réfrigératcurs ménagers de marque "Frigidaire " grand modèle, a été reproduit en un petit nombre d'exemplaires et expérimenté en A.O.F. et A.E.F. Comme le prototype $\mathrm{n}^{0} 1$, il comprenait une fabrique de glace $(4,500 \mathrm{~kg}$ de glace en cubes par cycle de 2 h à 2 h 30) et en plus un casier

(1) Établissements Zhendre, Direction Générale et 'Technique, 71-73, rue Abadie, Bordeaux. 
de conservation à $+5^{\circ}$, d'un volume de $16 \mathrm{dm}^{3}$. Son poids (135 kg) et son encombrement (longueur $110 \mathrm{~cm}$, largeur $67 \mathrm{~cm}$, hauteur $78 \mathrm{~cm}$ ) étaient très acceptables. Equipée d'un compresseur Everest type B.F.S 50 utilisant du Fréon 12, produisart à $-10^{\circ}+25^{\circ}$ et à 500 tours/minute $550 \mathrm{Fgh}$ et au régime d'utilisation $-15^{\circ}+50^{\circ}$ $360 \mathrm{Fgh}$, la machine était calculée pour fonc- même quantité de glace était obtenue, mais en un temps nettement plus court ( $2 \mathrm{~h}$ à $2 \mathrm{~h} 30)$. Les mêmes résultats furent enregistrés en brousse et la machine à glace idéale pour équipes mobiles d'immunisation semblait être trouvée. Malheureusement les canalisations de fréon s'avérèrent rapidement incapables de résister aux secousses et aux vibrations imposées à la machine par le

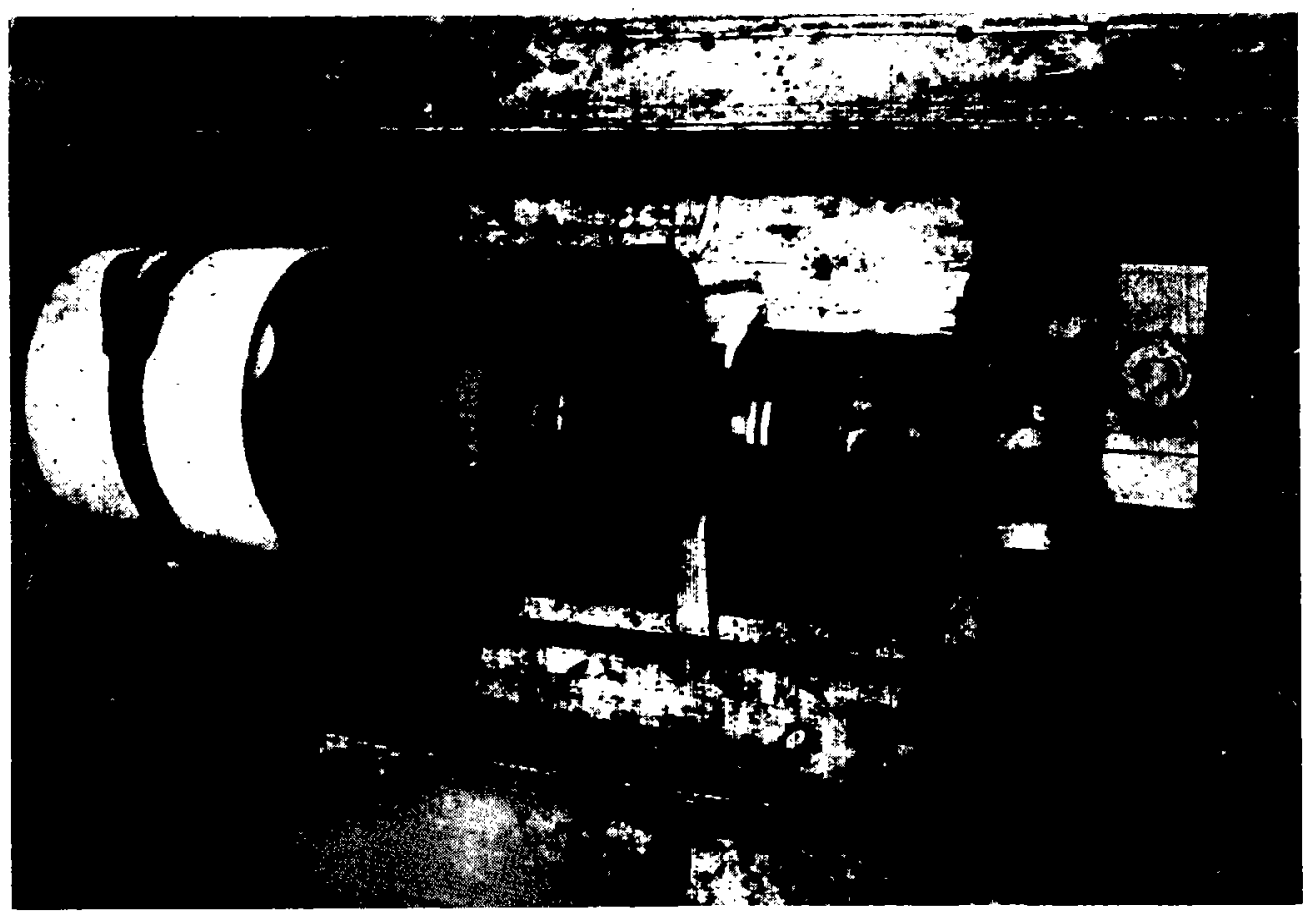

2. (Cliché A. Lalanne.) - Vue supérieure de l'appareil.

tionner à des températures ambiantes de l'ordre de $+45^{\circ}$, couramment enregistrées en zone sahélienne pendant la saison chaude. Une variante permettait, par addition d'un tiroir à glace supplémentaire, d'obtenir $6 \mathrm{~kg}$ de glace par cycle, le volume du casier de conservation étant alors porté à $20 \mathrm{dm}^{3}$. Dans tous les cas l'entraînement était réalisé avec un moteur Bernard à essence type $\mathrm{W} 9$, puissance $2 \mathrm{CV}$ à 3.000 tours minute.

J'ai personnellement expérimenté les prototypes I et II au Soudan Français (A.O.F.) en 1952-1954. A Bamako, par des températures de +38 à $+40^{\circ} \mathrm{C}$, on obtenait effectivement $4,500 \mathrm{~kg}$ de glace en 3 heures environ au cours du premier cycle, l'intérieur de l'appareil étant à la température ambiante. Si un deuxième cycle suivait immédiatement le premier, une déplacement des véhicules en tous terrains. Aucun remède valable n'a été trouvé jusqu'à ce jour et il a fallu, bien à regret, renoncer à cet appareil.

Arrivé à Madagascar en 1955, je retrouvai avec l'intensification de la lutte contre la maladie de Teschen, le même problème - non résolu le vaccin anti-Teschen devant être obligatoirement conservé à $+4^{\circ}$. Tous les appareils expérimentés jusqu'alors ayant péché par les canalisations, je songeai finalement à utiliser les " boules Singrün ", que javais remarquées dans certains postes isolés d'A.O.F., leur fonctionnement étant assuré de façon parfois surprenante pendant les années difficiles 1939-1945 (à bras, à bicyclette, la chaîne entrainant dans ce cas l'arbre de l'appareil au lieu de la roue arrière, etc.), mais toujours avec le même succès. 
Certes la quantité de glace obtenue par cycle serait moindre qu'avec les machines antérieurement expćrimentées, il n'y aurait plus de case de conservation à $+5^{\circ}$, mais après les échecs précédemment enregistrés, ce système paraissait avoir un avantage considérable du fait de l'absence de toute tuyauterie extérieure, les échanges gazeux se faisant à l'intérieur même de l'axe reliant les deux boules. par rapport au moteur Bernard de $2 \mathrm{CV}$. Mais ce moteur n'a pas donné entière satisfaction pendant la période d'essais en brousse et il lui a été substitué un moteur Bernard identique à celui équipant les deux premiers prototypes, mais placé en bout d'arbre, à commande directe, avec démultiplicateur et embrayage incorporés, la transmission par courroie étant ainsi supprimée.

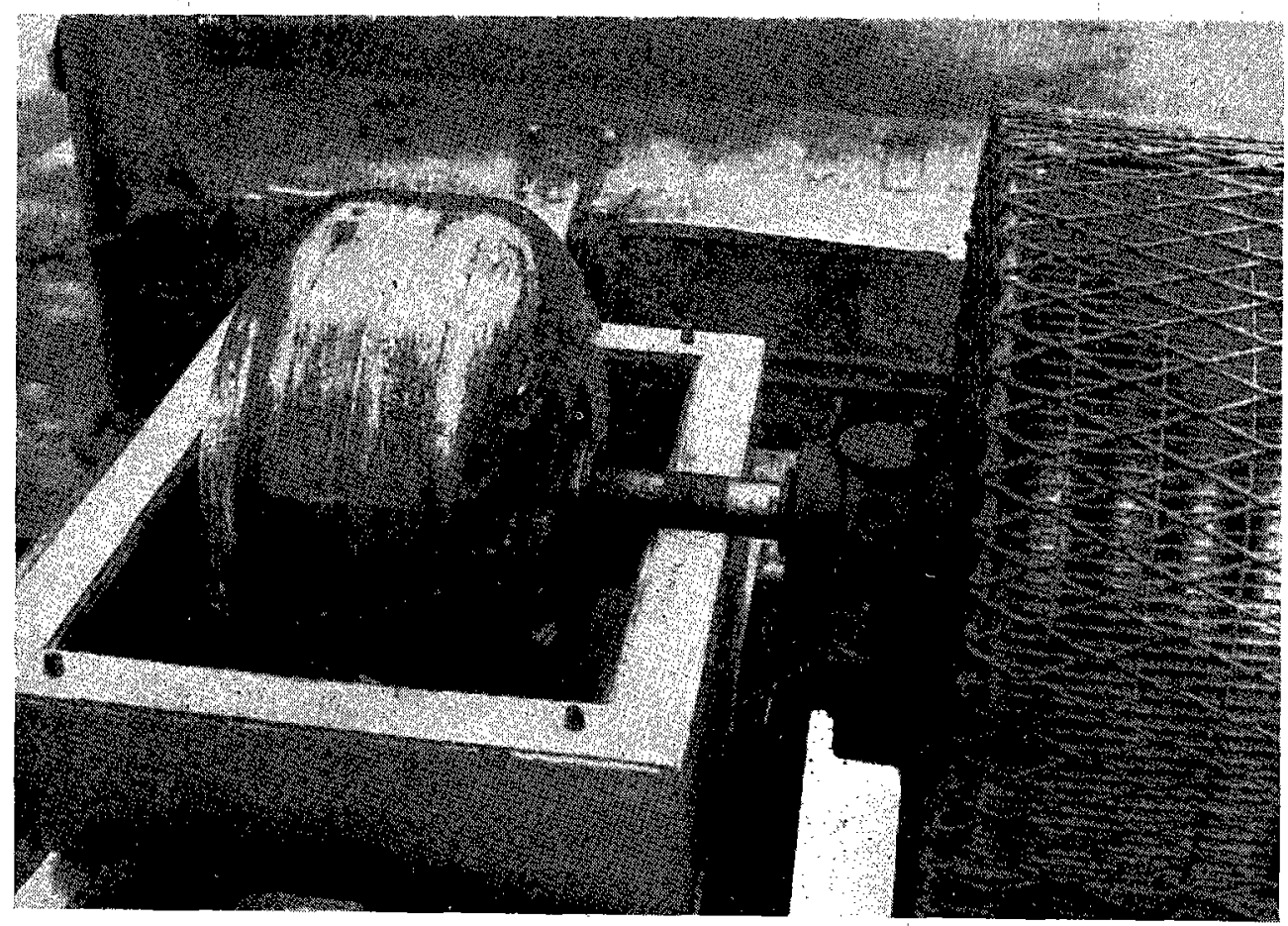

3. (Cliché A. Lalanne.) - L'opération est terminée, une calotte de glace recouvre la boule réfrigérante.

C'est ainsi qu'est né le troisième prototype de machine à glace pour équipes mobiles d'immunisation. Construit suivant mes indications, il a été mis gratuitement à ma disposition par le constructeur (2) pour expérimentation et essais dans les conditions de la pratique courante. Il comprend un groupe frigorigène A.S. (du nom des inventeurs Audiffren-Singrün), entraîné primitivement par un moteur à essence VAP de $1 \mathrm{CV}$ environ à 1.500 tours/minute (entraînement par courroie trapézoïdale), proposé par le constructeur en raison de son prix avantageux

(2) Société d'Applications Frigorifiques. Siège Social : 92, rue de la Victoire, Paris (IX ${ }^{\mathrm{e}}$ ). Direction générale et usines : 26, boulevard Michelet, Marseille (VIIIe). Que son Directeur trouve ici l'expression de nos bien vifs remerciements.

\section{Note technique sur le " Frigorigène A.S. "}

Le constructeur fournit sur le " Frigorigène A.S. " les indications suivantes :

"Le " Frigorigène A.S. " est un appareil entièrement hermétique à moteur indépendant, qui ne comporte ni joints, ni garnitures, ni presseétoupe et pour lequel toute fuite de gaz est supprimée.

" La charge initiale en gaz et huile est faite en usine lors de sa construction. Les recharges périodiques que nécessitent toujours les compresseurs ordinaires sont entièrement supprimées. Le poids constant du fluide réfrigérant en circuit assure à l'appareil une production invariable, quelle que soit son ancienneté. 
"Le " Frigorigène A.S. " est enfin caractérisé par la suppression pratique de l'usure. En effet, tous les organes en mouvement sont baignés par une huile spéciale inaltérable qui, travaillant en vase clos, conserve indéfiniment ses propriétés lubréfiantes. Cette suppression de l'usure est concrétisée pratiquement par la
" Le mécanisme intérieur se compose, côté condenseur, d'un carter et d'un couvercle de carter suspendus sur l'arbre et portant à leur base un contrepoids en plomb maintenant l'ensemble dans la position verticale au repos et dans une position stable et voisine de la verticale en période de marche.

\section{4 coupe du frigorigene (A)-S}

\section{(daprès le prospectus du constructeur)}

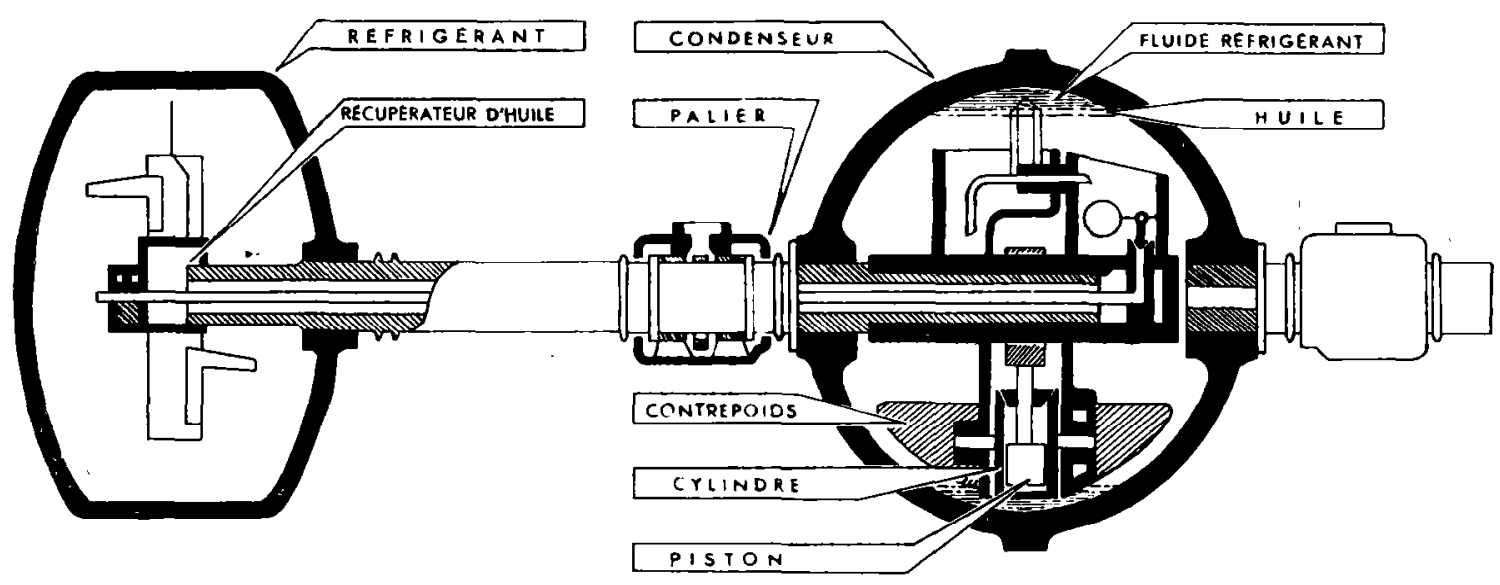

suppression de tous les organes de rattrapage de jeu, c'est-à-dire par la suppression des segments sur les pistons ou des garnitures aux tiges de pistons.

". Le "Frigorigène A.S. " se compose extérieurement de deux boules en bronze vissées et soudées réunies par un arbre creux, lui-même vissé et soudé sur les boules. Cet arbre est fermé à son extrémité après chargement par trois obturateurs dont un est soudé. L'ensemble est essayé sous l'eau à $14 \mathrm{~kg}$ de pression pour vérifier l'étanchéité absolue soit de la matière, soit des soudures. Il ne peut se produire par la suite de fuites de gaz ou des rentrées d'air lorsque le réfrigérant travaille à une pression inférieure à la pression atmosphérique (cause fréquente d'avaries pour les compresseurs frigorifiques ouverts du type courant).
“ Dans ce carter sont logés deux cylindres à double effet avec pistons sans segments, montés d'un seul bloc, avec leurs bielles de commande terminées par des colliers d'excentriques. Un excentrique est fixé sur l'arbre central. Les cylindres comportent chacun deux lumières d'aspiration en contact étroit avec les lumières correspondantes du couvercle du carter. Par différents canaux, ces lumières communiquent avec l'arbre creux et, par là, avec le réfrigérant.

"Chaque cylindre comporte aussi les lumières de refoulement fermées par des "reniflards", simples lames de ressort non ajourées travaillant sur une butée cylindrique et d'une résistance à toute épreuve.

" Enfin, à la partie haute du couvercle de carter se trouve la "boîte à flotteur " qui règle 
l'admission du fluide au réfrigérant par l'intermédiaire d'un tube calibré passant au centre de l'arbre creux.

"La boule réfrigérante ne renferme qu'un " récupérateur d'huile ", genre de vase Florentin, simplement suspendu qui ramène au condenseur les particules d'huile pouvant être entrainées au réfrigérant.

"Fonctionnement. En régime de marche, le fluide réfrigérant sous pression est projeté sur le grand diamètre du condenseur et se liquéfie au contact des parois froides de celui-ci. L'huile projetée elle aussi se sépare par différence de densité. Une " raclette" ramène le fluide dans une boîte de séparation qui communique par un siphon avec la boîte à flotteur. Les variations de niveau du gaz liquéfié permettent au flotteur de se soulever plus ou moins et, par l'intermédiaire du tube central, de régler l'admission au réfrigérant où s'opère la détente.'

" La dépression du réfrigérant est assurée par les cylindres et les différents canaux d'aspiration. Le gaz à l'état normal retourne aux cylindres où il est comprimé; rejeté dans le condenseur, projeté sur le grand, diamètre et liquéfié à nouvcau. Le cycle ainsi schématisé se reproduit indéfniment avec réglage automatique du circuit.

"Le danger de surpression est en marche totalement supprimé, car si la pression intérieure du condenseur atteint une valeur déterminée, le contrepoids devient insuffisant et l'ensemble, carter, cylindres, pistons est entraîné en un même mouvement. N'ayant plus de mouvement relatif entre pistons et cylindres, la pression se stabilise aussitôt.

"En d'autres termes, l'appareil de sécurité est en réalité la pesanteur qui ne peut jamais faire défaut et qui assure au "Frigorigène A.S. " la sécurité de marche absolue.

Un prospectus relatif au "Frigorigène A.S. " précise que cet appareil est :

"Rationnel. Le "Frigorigène A.S. ", appareil frigorifique hermétique de conception extrêmement rationnelle, convient à toutes les applications commerciales ou industrielles du froid.

"Simple. Le montage du "Frigorigène A.S. " n'exige aucun spécialiste et il peut fonctionner quelle que soit la force motrice utilisée, son moteur étant complètement indépendant.

" Etanche. " Le Frigorigène A.S. " ne nécessite aucune préparation pour la mise en route, aucune recharge de liquide réfrigérant, celui-ci étant introduit une fois pour toutes à l'usine au moment de la fabrication de l'appareil. "Le Frigorigène A.S. " ne comportant aucun joint, presse-étoupe ou garniture, aucune fuite n'est possible et son rendement reste constant quelle que soit son ancienneté.

"Sans entretien. L'entretien mécanique de l'appareil est pratiquement nul puisqu'il se résume au seul graissage des deux paliers extérieurs qui le supportent. Le "Frigorigène A.S. " ne connaît pas l'usure, son mécanisme intérieur baignant dans une huile spéciale qui, en vase clos, conserve indéfiniment ses qualités lubréfiantes.

" D'une grande sécurité. Les utilisateurs du froid connaissent les inconvénients qu'entraîne l'arrêt, même momentané, d'un appareil frigorifique, et les pertes subies par l'altération des marchandises qui en résultent. Les appareils. frigorigènes installés depuis 50 ans, bien que ne bénéficiant pas des derniers perfectionnements. techniques, fonctionnent encore aujourd'hui a la complète satisfaction d'une nombreuse clientèle, sans avoir subi aucune révision, ni réparation. On peut affirmer que l'emploi du "Frigorigène A.S. " offre une sécurité inconnue ailleurs".

\section{Groupe mobile à fabrication directe de glace}

Le constructeur présente deux types d'appareils : le "Frigorigène A.S. " proprement dit, avec condenseur à eau et réfrigérant à saumure pour installations fixes et l' "Aérotor A.S. ", avec condenseur à air et réfrigérant sur lequel se forme directement la glace, plus spécialement adapté aux installations mobiles. C'est l" "Aérotor A.S. " qui a été utilisé pour le montage du "groupe mobile à fabrication directe de glace". Moteur Bernard (avec embrayage et réducteur) et "Aérotor A.S. " ont été installés sur un berceau composé de deux robustes fers à $U$ de $8 \mathrm{~cm}$, pourvus d'une poignée à chaque extrémité pour faciliter les manipulations.

D'un poids à peine inférieur à $200 \mathrm{~kg}$, cette machine à glace mésure $1,485 \mathrm{~m}$ de long et $0,545 \mathrm{~m}$ de large. Sa hauteur atteint $0,55 \mathrm{~m}$ au niveau du réservoir à essence et $0,50 \mathrm{~m}$ au niveau du bac à eau. Malgré son poids relativement élevé, 4 hommes la manipulent aisément. Pour le transport, elle est simplement posée dans la caisse du pick-up, dans le sens de la longueur. Elle est bloquée de chaque côté 
par un morceau de madrier et l'ensemble est protégé par une solide caisse sans fond (emploi de l'emballage d'origine) pesant $40 \mathrm{~kg}$ et mesurant extérieurement $1,68 \mathrm{~m}$ de long, $0,65 \mathrm{~m}$ de large et $0,62 \mathrm{~m}$ de haut. De cette façon, tous les organes de la machine sont parfaitement à l'abri et le matériel de l'équipe mobile, ainsi que les bagages du personnel, peuvent être facilement placés à l'intérieur du véhicule, de part et d'autre et au-dessus de la machine à glace. Quant au personnel, réduit en général à 3 personnes (le chef de l'équipe mobile conduit lui-même le véhicule et ne dispose pas d'un chauffeur), il s installe dans la cabine et voyage ainsi très confortablement.

\section{Résultats pratiques}

Mise en service début mars 1958 dans un district voisin de Tananarive (Ambohidratrimo) pendant une semaine pour essais préliminaires, la machine fut ensuite remise à une équipe mobile dirigée par un contrôleur d'élevage autochtone, et chargée d'opérer dans le district d'Ankazobe (province de Tananarive), du 10 mars au 29 avril, suivant un programme étudié au cours des années antérieures et maintenant parfaitement au point.

Les interventions d'une équipe mobile sont en général multiples et portent sur la vaccination des porcs contre la maladie de Teschen, la vaccination des chiens et chats contre la rage (vaccin Flury), la vaccination des volailles contre la maladie de Newcastle, le choléra et la variole, la castration et le traitement contre les affections parasitaires internes des porcs, etc. Toutefois leur cadence est quelque peu ralentie du fait que la vaccination anti-teschen, qui nécessite 2 injections de vaccin, oblige l'équipe à passer deux fois dans les mêmes villages à 15 jours $d^{\prime}$ intervalle. Cette $2^{e}$ visite est mise à profit pour renouveler les traitements antiparasitaires internes.

En général, pour des raisons de commodité, l'équipe s'installe au chef-lieu de canton, où elle revient chaque soir, se rendant dans la joumée dans les villages environnants. La machine à glace est déposée dans un abri du campement ou chez le chef de canton. Elle y reste jusqu'au départ pour le canton suivant et fonctionne chaque jour autant de fois qu'il est nécessaire.

Les vaccins sont conservés dans des boîtes isothermes dont on trouve actuellement des modèles variés dans toutes les maisons spécia- lisées (coffres éboneige, isobox, etc.). Celles spécialement fabriquées pour le transport dans la métropole du vaccin contre la fièvre aphteuse donnent toute satisfaction outre-mer (solidité, légèreté, conservation de la glace, volume, etc.). Leur nombre et leur capacité sont en rapport avec la quantité de vaccin à conserver, les couches de récipients à vaccin alternant avec des couches de glace. Les bouteilles à glace, genre thermos (en verre, avec armature métallique), qui ont l'avantage de conserver plus longtemps la glace ont été cependant abandonnées en raison de leur trop grande fragilité et de leur prix élevé.

Chaque soir, à son retour au campement, l'équipe remplace la glace manquante, renouvelle l'opération le lendemain matin, si besoin est, avant son départ et emporte une petite réserve pour la journée. Les besoins journaliers sont de l'ordre de $10 \mathrm{~kg}$ pour un stock de vaccin de 3 à 4 semaines (1). Cette quantité est obtenue, dans la zone des plateaux, par des températures de l'ordre de $25^{\circ}$ sous abri, en 2 heures environ, en 4 cycles consécutifs : le jer d'une durée de 35 minutes, les autres légèrement plus courts ( 25 à 30 minutes). Le $1^{\text {er }}$ cycle donne $1,800 \mathrm{~kg}$ à $2 \mathrm{~kg}$ de glace et les suivants, qui bénéficient du refroidissement de l'ensemble (boule réfrigérante, bac à eau, eau disponible après le $1^{\text {er }}$ cycle), près de $3 \mathrm{~kg}$ (en moyenne $2,800 \mathrm{~kg}$ ). Il faut d'autant plus de temps, précise le constructeur, que la température ambiante est plus élevée. Le contrôle effectué sur la côte Ouest, dans la région de Majunga, où les températures de $35^{\circ}$ sont courantes en saison chaude, a donné les résultats suivants :

Température de l'air sous abri : $33 / 35^{\circ}$; Hygrométrie relative : 60 à $70^{\circ}$;

Température de l'eau du bac : 30 à $33^{\circ}$;

Durée du cycle : 40 minutes.

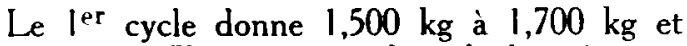
les suivants (l'eau restant dans le bac étant à la température de 4 à $\left.5^{\circ}\right), 2,600 \mathrm{~kg}$, soit une quantité très légèrement inférieure pour un temps de fonctionnement à peine plus long.

L'expérimentation de la machine n'a pu être faite par des températures plus élevées, celles habituellement observées en zone sahélienne africaine pendant la saison chaude $\left(40 / 45^{\circ}\right.$ sous abri), étant pratiquement inconnues à Madagascar. Cependant il est vraisemblable que cette

(1) Lc ravitaillement de l'équipe en vaccins est fait en général par le chef de la circonscription d'Elevage, à l'occasion de ses tournées d'inspection, toutes les trois à quatre semaines. 
machine donnera satisfaction même dans les pays les plus chauds. Il suffira de fabriquer la glace au moment le plus favorable, c'est-à-dire le matin de très bonne heure, la température dépassant rarement à ce moment $35^{\circ}$, ce qui nous rapproche des conditions rencontrées dans la région de Majunga.

Du restc il a été observé que la production de glace est influencée beaucoup plus par la

\section{Fonctionnement de la machine}

La mise en marche de cette machine et son fonctionnement sont d'une très grande simplicité. Il suffit de verser 5 litres d'eau dans le bac, de mettre le moteur en route, d'embrayer et de régler la vitesse suivant les indications du constructeur (les boules doivent tourner dans un sens déterminé, indiqué par une flèche, à 380 tours/minute, ce qui correspond, théori-

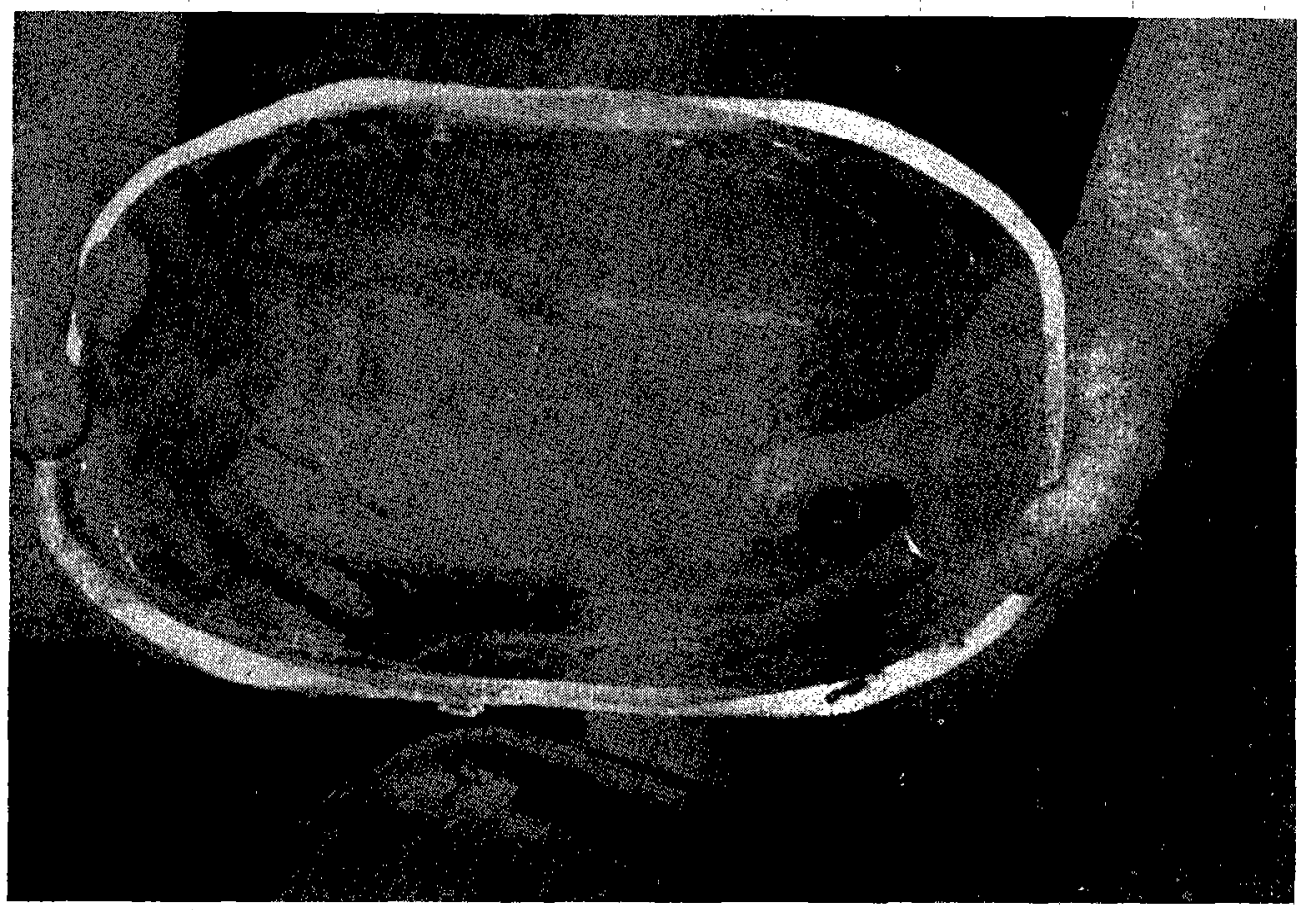

5. (Cliché R. Armaing.) - Une demi-calotte a été enlevée et la boule a effectué le demi-tour qui permettra d'enlever facilement la deuxième calotte.

température de l'eau versée dans le bac que par la température extérieure. C'est ainsi qu'on a obtenu, au cours d'un premier cycle, pour un même temps de rotation (40 minutes) et une même température extérieure $\left(33\right.$ à $\left.34^{\circ}\right): 1,770 \mathrm{~kg}$ de glace avec de l'eau à $30^{\circ}$ et $2,150 \mathrm{~kg}$ avec de l'eau à $24^{\circ}$, puisée à une source. Ceci est intéressant pour la zone sahélienne, où on peut facilement obtenir, par évaporation, un abaisscment de température de quelques degrés, l'eau étant placée dans des bidons métalliques de faible contenance ( 2 à 5 litres) entourés de linges humides ou mieux encore, dans des peaux de bouc exposées à l'air. quement, ̀̀ environ 1.200 tours/minute au moteur (1). Au début, un compte-tour de poche est nécessaire pour obtenir la vitesse optima (2), mais par la suite le réglage se fait avec une précision suffisante soit d'après le'bruit de l'échappement, soit d'après la position de l'accélérateur $\left(2^{\mathrm{e}}\right.$ ou $3^{\mathrm{e}}$ cran).

(1) Dans la pratique les meilleurs résultats ont été obtenus en faisant tourner le moteur aux environs de 1250 tours/minute.

(2) $\mathrm{Si}$ la vitesse de rotation des boules est trop élevée, l'eau est projetée à l'extérieur du bac et la glace se forme très irrégulièrement sur la boule réfrigérante. 
Pour l'arrêt, il faut réduire les gaz, débrayer et stopper le moteur.

Le prélèvement de la glace se fait de la façon suivante : après avoir enlevé le couvercle du bac réfrigérant, on sectionne, à l'aide d'un outil un peu tranchant, la calotte de glace en deux parties égales par une saignée horizontale. On enlève la demi-calotte supérieure qui a eu le temps de se décoller pendant cette opération.

Puis on fait faire un demi-tour à l'appareil (toujours dans le sens indiqué). Au bout de quelques minutes la deuxième demi-calotte se décolle d'elle-même.

Le décollage des demi-calottes de glace se fait par fusion partielle da le glace au contact de la boule réfrigérante réchauffée intérieurement par les gaz chauds venant du condenseur aussitôt après l'arrêt. Mais ce réchauffage n'est rapide que dans la partie supérieure du réfrigérant car la partie inférieure est en contact avec le liquide intérieur qui se réchauffe beaucoup plus lentement. C'est pour cette raison qu il faut faire faire un demi-tour à l'appareil pour enlever la deuxième calotte de glace.

Le sens de rotation est celui des aiguilles d'une montre pour un observateur placé près du moteur et regardant l'aérotor.

Consommation et prix de revient de la glace

Pour une vitesse de 1.250 tours/minute et une température de $33 / 35^{\circ}$ (région de Majunga), c'est-à-dire dans les conditions les plus défavorables à Madagascar, la consommation d'essence (valant $40 \mathrm{frs}$ le litre) est de $0,600 \mathrm{l} /$ heure. La production des $10 \mathrm{~kg}$ de glace nécessaires chaque jour, obtenus en quatre cycles consécutifs d'environ 40 minutes chacun, revient donc à $64 \mathrm{frs}$, soit 6,40 frs le kg.

Outre la dépense d'essence, il y a lieu de tenir compte également dans le calcul du prix de revient de la glace, de l'amortissement de la machine (calculé sur 10 ans, prix de la machine $200.000 \mathrm{frs}$, soit $20.000 \mathrm{frs}$ par an) de son entretien (outre le renouvellement de l'huile du moteur et le graissage des paliers, qui représentent une dépense négligeable, il se limite pratiquement au remplacement des pièces essentielles du moteur Bernard, voire du moteur lui-même, tous les 4 à 5 ans, soit une dépense de $6.600 \mathrm{frs}$ ) et, enfin, de l'intérêt de la somme engagée ( $10.000 \mathrm{frs}$ par an à $5 \%$ o. La totalité de ces frais s'élève à $36.600 \mathrm{frs}$ par an, soit $100 \mathrm{frs}$ par jour, ou encore $10 \mathrm{frs}$ par $\mathrm{kg}$ de glace.
En définitive le prix de revient de la glace est de 16,40 frs le $\mathrm{kg}$ (essence 6,40 frs, frais généraux $10 \mathrm{frs}$ ).

\section{Entretien de la machine à glace}

Le mécanisme intérieur ne demande aucun entretien puisqu'il est entièrement enfermé. Il faut simplement graisser les paliers extérieurs, mais cette opération doit être soigneusement effectuée avant chaque mise en marche (huile SAE 30) car le défaut de graissage entrainerait l'usure rapide de l'arbre qui, étant creux ne peut supporter une grosse usure sans risque de rupture.

Lorsque l'appareil fonctionne dans des conditions normales (nombre de tours, sens de la rotation, température de l'air et de l'eau utilisée pour la fabrication de la glace), on ne doit constater pratiquement jamais d'avaries du mécanisme intérieur. Si elles se produisaient, aucune réparation ne pourrait être effectuée sur place et le renvoi de la machine à l'usine serait indispensable. Toute diminution de production doit donc être attribuée, en principe, à une cause extérieure.

A noter que le condenseur de l'aérotor doit être bien aéré, sinon l'air s'échauffe et le rendement de l'appareil diminue sensiblement.

\section{Prix de la machine à glace}

Le prix de revient de la machine à glace dans les Territoires d'Outre-Mer n'est pas encore exactement connu, mais il sera, vraisemblablement, de l'ordre de 200.000 francs CFA.

On aurait pu réduire sensiblement ce prix en remplaçant le moteur Bernard par un système moins onéreux d'entraînement de l'aérotor (entrainement à bras, ou avec la prise de force arrière du pick-up Land-Rover). Mais le moteur Bernard, connu pour sa sobriété et sa rusticité, $\mathrm{m}$ a paru, en définitive, préférable à tous les autres systèmes, parce que plus pratique et plus efficace.

\section{Conclusion}

Une machine à glace transportable, spécialement conçue pour les équipes mobiles d'immunisation, composée d'un "Aérotor A.S. " entraîné par un petit moteur Bernard, a donné toute satisfaction au cours d'essais d'une durée de deux mois, effectués dans les conditions normales 
d'utilisation en brousse. La quantité de glace nécessaire chaque jour pour la conservation du vaccin est produite rapidement et avec la plus grande facilité. Cet appareil nouveau, dont l'équivalent n'existait pas encore sur le marché, extrêmement robuste, en même temps que très simple, pouvant être mis à la disposition d'un personnel non spécialisé, permet enfin de résoudre économiquement le problème du ravitaillement en glace des équipes mobiles d'immunisation opérant dans les territoires sous-développés.

(Service de l'élevage et des épizooties de Madagascar.)'

\section{SUMMARY}

\section{A portable ice-machine devised for immunisation mobile units}

A portable ice-machine devised especially for rinderpest immunisation mobile units, has given every satisfaction during trials over 2 months, carried out under the normal conditions of work in the "bush ". The amount of ice required to conserve the virus-vaccine is produced easily and quickly.

This new apparatus which has not appeared previously on the market is very robust, and at the same time simple to work by non-specialist native personnel. Its application will resolve the problem economically, of supplying ice to mobile units in under-developed countries.

\section{RESUMEN}

\section{Una máquina de hielo para equipos móviles de immunización}

Una máquina de hielo portátil, especialmente concebida para los equipos móviles de immunización ha dado entera satisfacción en el curso de ensayos de una duración de dos meses, efectuados en las condiciones normales de utilización. La cantidad de hielo necesaria cada dia para la conservación de la vacuna es producida rápidamente y con la mayor facilitad. Este aparato nuevo, cuyo equivalente no existía aún en el mercado, extremadamente robusto, al mismo tiempo que muy simple, pudiendo ser puesto a la disposición de un personal no especializado, permite al f́rn resolver económicamente el problema del aprovisionamiento en hielo de los equipos móviles de immunización que operan en los territorios subdesarrollados. 